\title{
Significant Benefits of AIP Testing and Clinical Screening in Familial Isolated and Young-onset Pituitary Tumors
}

\author{
Pedro Marques, ${ }^{1}$ Francisca Caimari, ${ }^{1}$ Laura C. Hernández-Ramírez, ${ }^{1,2, \bullet}$ David Collier, \\ Donato lacovazzo, ${ }^{1}$ Amy Ronaldson, ${ }^{1}$ Kesson Magid, ${ }^{1}$ Chung Thong Lim, \\ Karen Stals, ${ }^{3}$ Sian Ellard, ${ }^{3}$ Ashley B. Grossman, ${ }^{1}$ and Márta Korbonits ${ }^{1}$ (on behalf of \\ the FIPA Consortium) \\ ${ }^{1}$ Centre for Endocrinology, William Harvey Research Institute, Barts and the London School of Medicine \\ and Dentistry, Queen Mary University of London, London, UK; ${ }^{2}$ Section on Endocrinology \& Genetics, \\ Eunice Kennedy Shriver National Institute of Child Health and Human Development (NICHD), National \\ Institutes of Health (NIH), Bethesda, Maryland 20892-1862; and ${ }^{3}$ Exeter Genomics Laboratory, Royal \\ Devon \& Exeter NHS Foundation Trust, UK
}

ORCiD numbers: 0000-0002-4959-5725 (P. Marques); 0000-0003-1176-6186(A. B. Grossman); 0000-0002-4101-9432 (M. Korbonits).

Context: Germline mutations in the aryl hydrocarbon receptor-interacting protein (AIP) gene are responsible for a subset of familial isolated pituitary adenoma (FIPA) cases and sporadic pituitary neuroendocrine tumors (PitNETs).

Objective: To compare prospectively diagnosed AIP mutation-positive (AIPmut) PitNET patients with clinically presenting patients and to compare the clinical characteristics of AIPmut and AlPneg PitNET patients.

Design: 12-year prospective, observational study.

Participants \& Setting: We studied probands and family members of FIPA kindreds and sporadic patients with disease onset $\leq 18$ years or macroadenomas with onset $\leq 30$ years $(n=1477)$. This was a collaborative study conducted at referral centers for pituitary diseases.

Interventions \& Outcome: AIP testing and clinical screening for pituitary disease. Comparison of characteristics of prospectively diagnosed $(n=22)$ vs clinically presenting AIPmut PitNET patients $(n=145)$, and AIPmut $(n=167)$ vs AIPneg PitNET patients $(n=1310)$.

Results: Prospectively diagnosed AIPmut PitNET patients had smaller lesions with less suprasellar extension or cavernous sinus invasion and required fewer treatments with fewer operations and no radiotherapy compared with clinically presenting cases; there were fewer cases with active disease and hypopituitarism at last follow-up. When comparing AIPmut and AIPneg cases, AIPmut patients were more often males, younger, more often had GH excess, pituitary apoplexy, suprasellar extension, and more patients required multimodal therapy, including radiotherapy. AlPmut patients $(n=136)$ with GH excess were taller than AIPneg counterparts $(n=650)$.

ISSN Print 0021-972X ISSN Online 1945-7197

Printed in USA

(C) Endocrine Society 2020.

This is an Open Access article distributed under the terms of the Creative Commons Attribution License (http://creativecommons.org/licenses/by/4.0/), which permits unrestricted reuse, distribution, and reproduction in any medium, provided the original work is properly cited.

Received 25 October 2019. Accepted 28 January 2020.

First Published Online 30 January 2020.

Corrected and Typeset 7 April 2020.

Abbreviations: AIP, aryl hydrocarbon receptor-interacting protein; AIPmut, AIP mutation-positive; FIPA, familial isolated pituitary adenoma; GH, growth hormone; IGF-1, insulin-like growth factor 1; MEN1, multiple endocrine neoplasia type 1; MRI, magnetic resonance imaging; NF, nonfunctioning; NS, not specified; PitNET, pituitary neuroendocrine tumor. 


\begin{abstract}
Conclusions: Prospectively diagnosed AIPmut patients show better outcomes than clinically presenting cases, demonstrating the benefits of genetic and clinical screening. AIP-related pituitary disease has a wide spectrum ranging from aggressively growing lesions to stable or indolent disease course. (J Clin Endocrinol Metab 105: e2247-e2260, 2020)
\end{abstract}

Key Words: pituitary neuroendocrine tumor, pituitary adenoma, familial isolated pituitary adenoma, somatotropinoma, gigantism, aryl hydrocarbon receptor-interacting protein

$\mathbf{P}$ ituitary neuroendocrine tumors (PitNETs) are relatively common $(\sim 1: 1000$ clinically relevant cases in the general population) and familial cases represent around $5 \%$ of this patient cohort $(1,2)$. Familial isolated pituitary adenoma (FIPA) is a heterogeneous condition that involves the presence of PitNETs in 2 or more members of the same family without other syndromic manifestations. Up to $20 \%$ of all FIPA and $50 \%$ of familial acromegaly kindreds carry germline mutations in the aryl hydrocarbon receptor-interacting protein $(A I P)$ gene $(1,3,4)$. These mutations are also seen in sporadically diagnosed PitNETs (simplex cases), particularly in young patients, where the lack of family history is usually due to incomplete penetrance rather than de novo mutations (5-8). The typical AIP mutation-positive (AIPmut) phenotype is characterized by a young patient presenting with a large invasive growth hormone $(\mathrm{GH})$-secreting PitNET that is refractory to conventional treatments $(1,3-5,9-11)$, with AIPmut somatotropinomas being responsible for $29 \%$ of pituitary gigantism cases (12).

Family members at risk of inheriting an AIP mutation are recommended to undergo genetic testing and carriers are referred for clinical screening of pituitary disease $(1,3,13-15)$. The rationale behind this strategy is that identifying PitNETs in AIPmut carriers with otherwise unrecognized disease at an early stage increases the likelihood of effective treatment and remission $(1,3,14)$. The assumption is that screeningdiscovered PitNETs (ie, prospectively diagnosed PitNETs) are diagnosed at a less advanced stage and are less invasive than PitNETs with a clinical presentation, and thus should show a more favorable response to treatment and better clinical outcomes. However, these predicted advantages have never been actually shown in a prospective study.

Here, we present the results of a 12-year follow-up study on a large cohort of AIPmut patients, where we have characterized prospectively diagnosed AIPmut PitNET patients compared with clinically presenting cases. Our results highlight the critical importance of AIPmut genetic screening in selected individuals, and of clinical follow-up in known AIPmut carriers. Furthermore, we have expanded the description of
AIPmut PitNET phenotype, disease course, and outcomes compared with AIPneg cases.

\section{Materials and Methods}

\section{Study population}

We selected our study population from our cohort (2079 patients with PitNETs and their 1029 unaffected relatives) recruited via the collaborative research network of the International FIPA Consortium (collaborators listed at the end of the manuscript) between February 2007 and April 2019; details of recruitment have been previously described (3). All participants gave written informed consent approved by the local ethics committee. Indications for AIP genetic testing were (1) patients with FIPA; (2) macroadenomas with disease onset at $\leq 30$ years; and (3) PitNETs with disease onset at $\leq 18$ years. First-degree family members of individuals carrying AIP mutations were offered genetic testing. We included in our analysis all patients with known AIP mutational status matching these criteria $(n=1477)$. We excluded patients with undetermined affected status (ie, proven AIPmut carriers who did not undergo clinical screening or had pending clinical test results by the time of data analysis). Patients with X-linked acrogigantism or syndromic disease (multiple endocrine neoplasia type 1 (MEN1), MEN4, Carney complex, SDHxrelated, McCune-Albright and DICER1 syndromes, identified on the basis of clinical, biochemical, and genetic testing as appropriate) were excluded.

Of 1477 patients included in the study, 167 were AIPmut (33 not reported previously (11)), 154 with documented germline AIP pathogenic/likely pathogenic variant, and 13 affected subjects with predicted AIPmut status (obligate carriers in AIPmut kindreds but not formally tested, including subjects already deceased). Pathogenicity of AIP variants was determined as previously described $(3,11)$; only pathogenic or likely pathogenic variants were considered to be "mutations". The AIPneg subgroup included 1310 patients with PitNETs in whom a germline AIP mutation was excluded by genetic testing of all simplex probands and of the youngest affected member in the families.

\section{Genetic testing and clinical screening}

$A I P$ testing was performed using either Sanger sequencing and multiplex ligation-dependent probe amplification, or targeted next-generation sequencing on genomic DNA obtained from blood or saliva samples $(3,11,16)$. All the unaffected individuals with positive genetic screening for AIP were advised to undergo clinical, biochemical, and image screening tests by their local physician for the early diagnosis of possible pituitary disease. Follow-up was advised on an annual basis or as appropriate $(1,3,13)$. 


\section{Study groups and clinical parameters}

The familial cohort comprised patients with FIPA. The sporadic cohort included patients with young onset PitNETs ( $\leq 30$ years) with no known family history of PitNETs or syndromic disease. The clinical diagnoses were established as $\mathrm{GH}$ excess (acromegaly and gigantism), prolactinomas, clinically nonfunctioning (NF)-PitNETs, Cushing's disease, and thyrotropinomas, as previously described (3). Cases where the diagnosis was not specified due to unavailability of histopathological, clinical, or biochemical data were termed "PitNET not specified (NS)". Age of onset was defined as the age of presentation of first symptoms. Macroadenomas were defined as tumor size $\geq 10 \mathrm{~mm}$. Hypopituitarism at diagnosis and at last follow-up was defined as the presence of at least 1 pituitary deficiency documented biochemically. The number of treatments included the number of individual treatments received (each medication, surgery, and radiotherapy). Multimodal treatment was defined as the employment of 2 or more distinct forms of treatment in patient management. The reoperation subgroup involved patients who had at least 1 additional surgery following their first operation. Active disease was considered in patients with secretory PitNETs displaying the respective pituitary hormone above the normal assay range, and/or evidence of persistent or recurrent progressive tumor remnants in the surveillance pituitary magnetic resonance imaging (MRI) scans for both secretory PitNETs and NF-PitNETs. Small persistent tumor remnants after operation, stable over a period of time, and requiring no further intervention were considered as not active NF-PitNETs.

\section{Statistical analysis}

Qualitative variables were expressed as percentages and analyzed with the $\chi^{2}$ test to compare 2 or more groups. Quantitative or continuous variables were tested for Gaussian distribution with the Shapiro-Wilk test, and nonparametric and parametric data were then further analyzed with the Mann-Whitney U and Student t-tests, respectively. $P<.05$ was considered statistically significant. Statistical analyses were carried out using the SPSS software version 20 (IBM, USA) and GraphPad version 6 (Prism, USA). Data are presented as mean and standard deviation for continuous variables and as percentages for categorical variables.

\section{Results}

\section{General characterization of the study population}

Of the 1477 patients with PitNETs, 167 were AIPmut $(11.3 \%)$, and 1310 were AIPneg patients (FIPA or age $\leq 30$ years at onset). Demographic and clinical characteristics and comparative analysis of AIPmut vs AIPneg PitNETs are presented in Table 1 and Fig. 1. The familial cohort (355 families, 700 patients, 47\% of the whole study population) consisted of 37 AIPmut kindreds (114 patients) and 318 AIPneg families (586 patients). Of the 37 AIPmut families, 36 (97.8\%) had at least 1 somatotropinoma case, 19 were homogeneous somatotropinoma kindreds, and 1 was homogeneous prolactinoma family. Of the 318 AIPneg families, 146 $(46 \%)$ were homogeneous and 172 were heterogeneous, with detailed subtypes shown in Table 2 . In the sporadic cohort $(\mathrm{n}=777), 53(6.8 \%)$ had an AIP mutation (Table 1). Within the sporadic tumor subgroup, $10.5 \%$ (50 out of 477 ) of somatotropinomas, $1.5 \%$ (3 out of 197) of prolactinomas, and none (0 out of 54) of the NF-PitNET cases were found to harbor a germline AIP mutation (Table 3; all supplementary material and figures are located in a digital research materials repository (17)).

\section{Prospectively diagnosed vs clinically presenting AIPmut PitNETs}

Genetic testing of AIPmut kindreds identified 187 apparently unaffected AIPmut carriers. A total 165 AIPmut carriers were disease free at both baseline screening and at last follow-up assessment (mean follow-up duration $5.9 \pm 3.3$ years, ranging between 1 and 11 years), while 22 subjects $(11.8 \%)$ were prospectively diagnosed with a PitNET. The mean age at diagnosis of prospectively diagnosed AIPmut PitNET patients $(30.4 \pm 15.7$ years $)$ and the age at genetic testing of unaffected AIPmut carriers $(35.9 \pm 24.1$ years $)$ did not differ $(P=.453)$. There was no significant difference in the gender distribution either: $49.7 \%$ prospectively diagnosed males vs $63.6 \%$ unaffected carrier males $(P=.219)$.

Three of these prospectively diagnosed cases had normal biochemistry and contrast-enhanced pituitary MRI scan at baseline screening, but went on to develop a PitNET during the subsequent follow-up: 2 small NF-PitNETs and 1 microprolactinoma, being stable since their initial detection and none requiring intervention to date. Eight of the 22 cases (36\%) had retrospectively recognized symptoms that could be attributed to pituitary disease. Prospectively diagnosed PitNETs were smaller than clinically presenting tumors $(10 \pm 7$ vs $24 \pm 13 \mathrm{~mm} ; P<.001)$, and $68 \%$ vs $8 \%$ were microadenomas $(P<.001$, Table 4 and Fig. 2A). Prospectively diagnosed PitNETs were associated with lower rates of hypopituitarism at diagnosis (0 vs $58 \% ; P<0.001)$, suprasellar extension $(11 \%$ vs $68 \% ; P<.001)$, and cavernous sinus invasion $(11 \%$ vs $44 \% ; P=.010$ ) (Table 4 and Fig. 2 A). Prospectively diagnosed cases required fewer treatments $(0.7 \pm 1.0$ vs $2.3 \pm 1.7 ; P<.001)$ and operations $(0.4 \pm 0.5$ vs $1.0 \pm 0.8 ; P<.001$ ), none received radiotherapy (vs $38 \% ; P<.001)$, and had decreased rates of active disease $(6 \%$ vs $28 \% ; P=.039)$ and hypopituitarism $(0$ vs $41 \% ; P=.003$ ) at last follow-up (Table 4 and Fig. $2 \mathrm{~B}$ and $2 \mathrm{C})$. 
Table 1. Characteristics of the study population and comparative analysis of AIPmut vs AIPneg patients

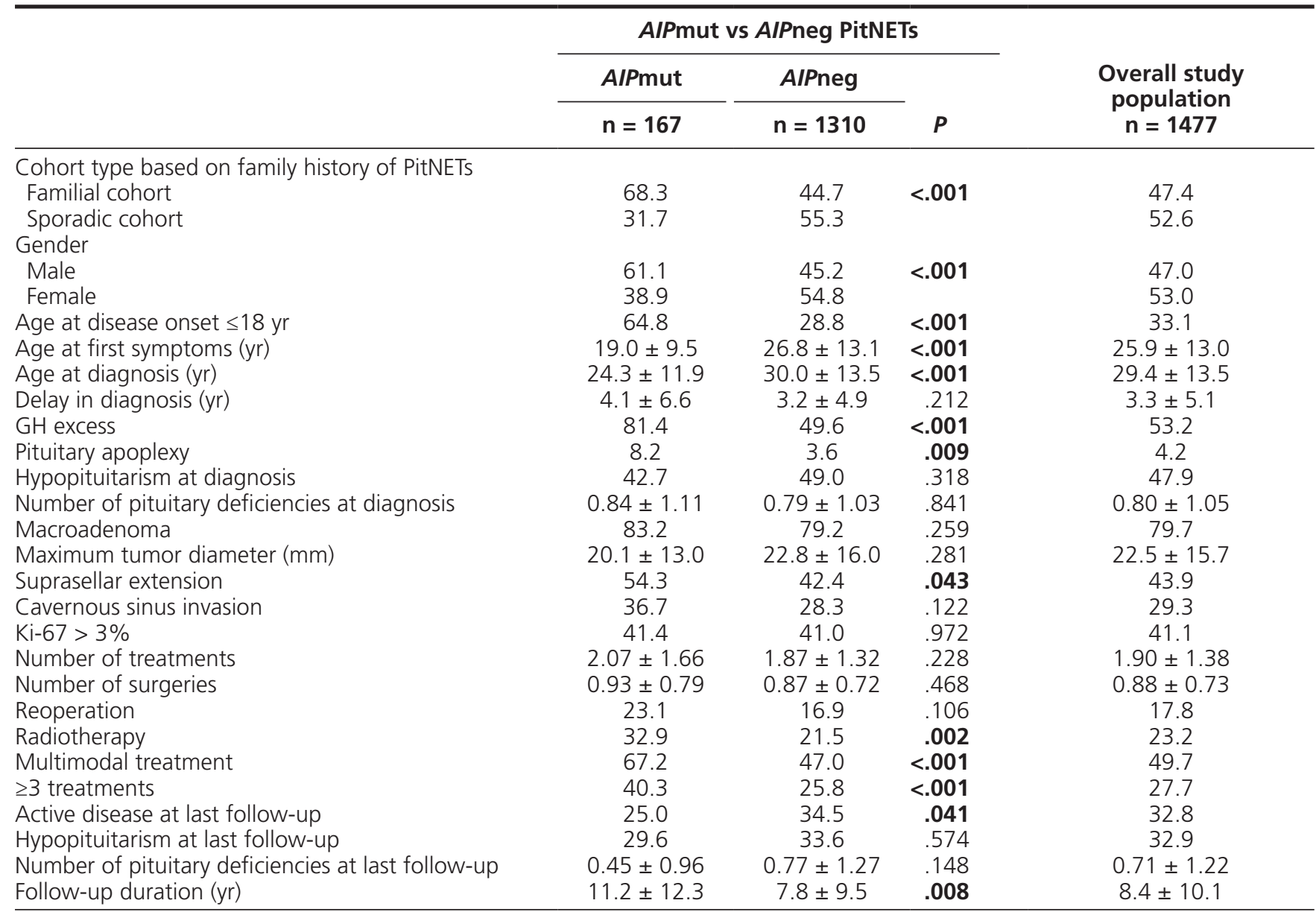

Categorical data are shown as \%; continuous variables are shown as mean \pm standard deviation. $P$ values in bold are those $<.05$ (statistically significant).

Abbreviations: AIPmut, AIP mutation-positive; AIPneg, AIP mutation-negative; GH, growth hormone; PitNET, pituitary neuroendocrine tumor; yr, years.

Prospectively diagnosed somatotropinomas, NF-PitNETs and prolactinomas had significantly lower rates of hypopituitarism at diagnosis, macroadenomas, and suprasellar extension, requiring fewer treatments than those clinically presenting counterparts (Table 4). Prospectively diagnosed AIPmut somatotropinomas were also significantly smaller and none required radiotherapy $(P=.009)$. None of the prospectively diagnosed AIPmut NF-PitNETs had hypopituitarism $(P=.002)$ or active disease $(P=.035)$ at last follow-up (Table 4). Two AIPmut patients had prospectively diagnosed microprolactinomas with no suprasellar extension or cavernous sinus invasion, and were eupituitary at diagnosis and at last follow-up: 1 responded well to dopamine agonist and the other is under observation (described in detail as case 5 in (14)).

\section{AIP mutations in the study population}

Forty-four different germline pathogenic/likely pathogenic AIP mutations were identified, including 5 previously not described mutations (exon 1 deletion; c.344delT
(p.L115fs*41); c.773T>G (p.L258R); c.779delA (p.K260fs*44); c.863_864del (p.F288Cfs*?)), among the 167 AIPmut patients (17). The most common mutation types were nonsense mutations $(27 \%)$ and frameshift mutations $(25 \%)$, followed by missense $(18 \%)$, splice site $(7 \%)$, in-frame insertions/deletions $(9 \%)$, and large genomic deletions ( $7 \%$ ), and we had 1 each of promoter, start site, and stop-loss mutations. Of 167 AIPmut PitNETs, $127(76 \%)$ were due to a truncating mutation, and the most frequent AIP mutation was c.910C >T (p.R304*), which was detected in 57 patients.

In our study population, we identified 17 different $A I P$ variants classified as benign, likely benign, or variants of uncertain significance according to the American College of Medical Genetics and Genomics and the Association for Molecular Pathology criteria $(17,18)$. We note that one of the most common AIP variants identified, p.R304Q, although controversial, is currently classified as variant of uncertain significance (19), patients from these kindreds were allocated to the AIPneg subgroup. 
A)

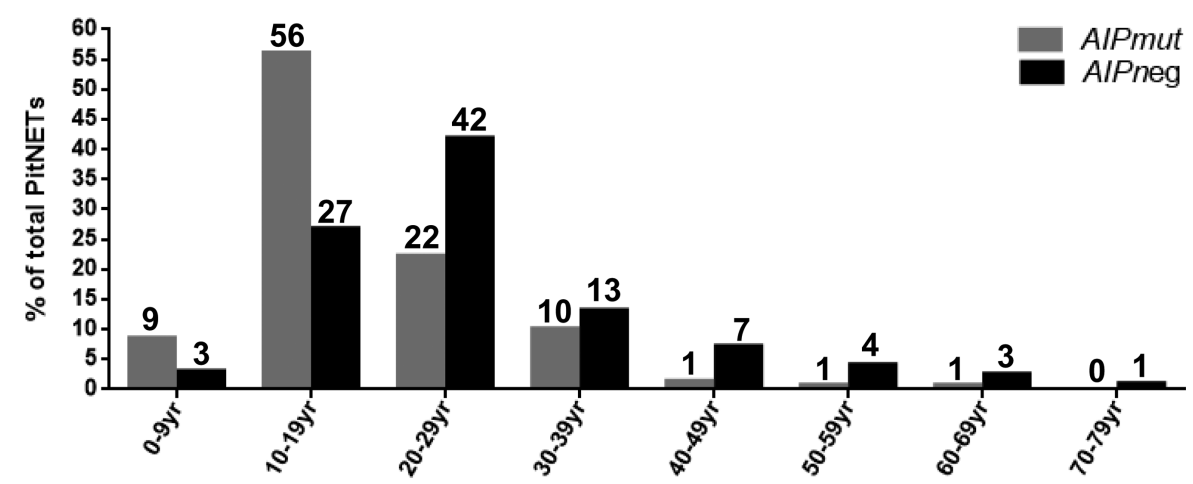

B)

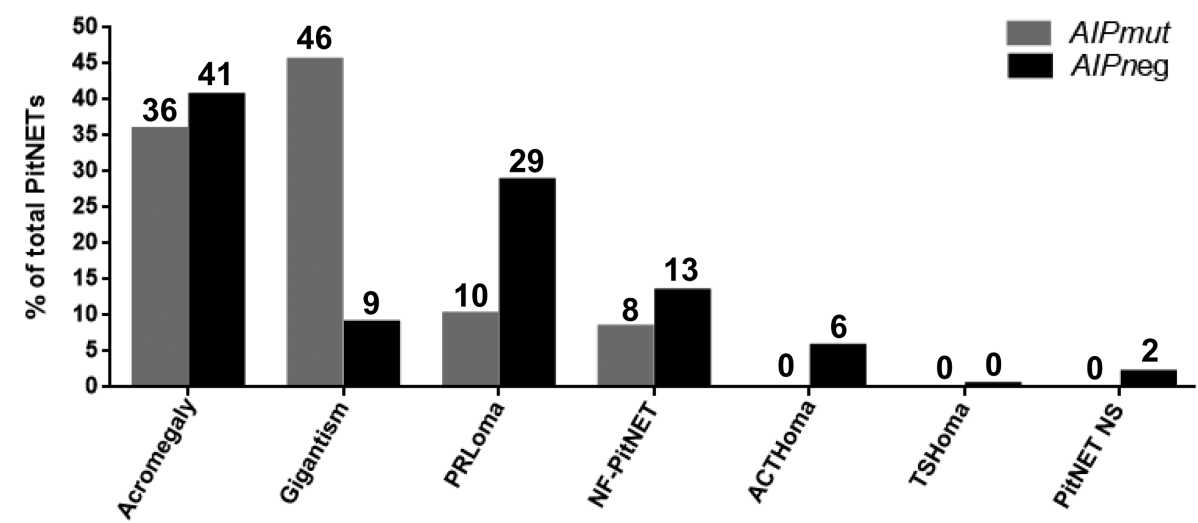

Figure 1. Distribution of AIPmut vs AIPneg PitNETs according to age at onset (A) and to clinical diagnosis (B). Numbers above columns represent percentage of patients. We note that the two AIPmut cases with first symptoms in the 5th and 6th decade, both had macroprolactinomas, 1 presenting with apoplexy. ACTHoma, ACTH-secreting adenoma or Cushing's disease; AIPmut, AIP mutation-positive; AIPneg, AIP mutationnegative; PitNET NS, pituitary neuroendocrine tumor not specified; NF-PitNET, non-functioning PitNET; PRLoma, prolactinoma; TSHoma, thyrotropinoma; yr, years.

\section{Comparative analysis of AIPmut vs AIPneg patients}

Overall, patients with AIPmut were more frequently males $(61 \%$ vs $45 \% ; P<.001)$ than AIPneg patients, 8 years younger at first symptoms, and 6 years younger at diagnosis, with disease onset $\leq 18$ years in $65 \%$ and $<30$ years in $87 \%$ (Table 1 and Fig. 1A). AIPmut PitNETs had a higher rate of pituitary apoplexy and suprasellar extension, more often required radiotherapy, and multimodal and multiple treatments than AIPneg ones (Table 1). Patients with AIPmut had lower rates of active disease at last follow-up (25\% vs $35 \% ; P=.041)$. However, as AIPmut had a longer follow-up, we analyzed only patients with no longer than a 10 year follow-up, and then there was no difference in the rate of active disease at last follow-up (39\% vs $43 \% ; P=.642$ ).

AIPmut PitNETs were more often associated with $\mathrm{GH}$ excess, with gigantism being the predominant clinical diagnosis (Fig. 1B and (17)). AIPmut patients with $\mathrm{GH}$ excess were younger than AIPneg cases (Table 3). There was no difference in insulin-like growth factor 1 (IGF-1) levels at diagnosis between patients presenting clinically with AIPmut and AIPneg $(P=.696$, Table 3$)$. All AIPmut somatotropinomas were sparsely granulated in contrast to $68 \%$ of the AIPneg ones $(P<.001)$; similar ratios were seen considering only $A I P$ mut and AIPneg giants. AIPmut somatotropinomas were associated with higher rates of pituitary apoplexy, suprasellar extension, radiotherapy, and reoperation, and showed trends for an increased need for multimodal therapy $(P=.076)$ and $\geq 3$ treatments $(P=.079)$. The mean final height was higher in the AIPmut somatotropinoma subgroup both for males $(193 \pm 18$ vs $185 \pm 14 \mathrm{~cm} ; P=.004)$ and females $(175 \pm 13$ vs $169 \pm 9 \mathrm{~cm} ; P=.017)$ (Table 3$)$. Patients with AIPmut prolactinomas had higher rates of pituitary apoplexy than AIPneg counterparts, which remained significantly higher when considering only clinically presenting cases (17). AIPmut NF-PitNETs had lower rates of macroadenomas, hypopituitarism at last follow-up, lower tumor diameter, and fewer pituitary deficiencies at diagnosis, as well as requiring fewer treatments and surgery than their nonmutated counterparts; however, when excluding the 10 prospectively diagnosed AIPmut NF-PitNETs patients these significant differences were lost ((17)). 
Table 2. AIPmut and AIPneg FIPA kindreds according to pituitary tumor types

\begin{tabular}{|c|c|c|c|}
\hline & AIPmut kindreds & AIPneg kindreds & Total \\
\hline ACTHoma only & 0 & $7(2.2)$ & $7(2.0)$ \\
\hline ACTHoma + GHoma & 0 & $7(2.2)$ & $7(2.0)$ \\
\hline ACTHoma + NF-PitNET & 0 & $1(0.3)$ & $1(0.3)$ \\
\hline ACTHoma + NF-PitNET + PitNET NS & 0 & $1(0.3)$ & $1(0.3)$ \\
\hline ACTHoma + PRLoma & 0 & $8(2.5)$ & $8(2.3)$ \\
\hline GHoma only & $19(51.4)$ & $68(21.4)$ & $87(24.5)$ \\
\hline GHoma + NF-PitNET & $8(21.6)$ & $25(7.9)$ & $33(9.3)$ \\
\hline GHoma + NF-PitNET + PRLoma & $1(2.7)$ & $4(1.3)$ & $5(1.4)$ \\
\hline GHoma + PitNET NS & 0 & $19(6.0)$ & $19(5.3)$ \\
\hline GHoma + PitNET NS + PRLoma & 0 & $1(0.3)$ & $1(0.3)$ \\
\hline PRLoma only & $1(2.7)$ & $47(14.8)$ & $48(13.5)$ \\
\hline PRLoma + FSHoma & 0 & $1(0.3)$ & $1(0.3)$ \\
\hline PRLoma + PitNET NS & 0 & $18(5.7)$ & $18(5.1)$ \\
\hline
\end{tabular}

Data are shown as $\mathrm{n}(\%)$.

Abbreviations: ACTHoma, ACTH-secreting adenoma or Cushing's disease; AlPmut, AIP mutation-positive; AIPneg, AlP mutation-negative; FSHoma, FSH-secreting adenoma; GHoma, GH-secreting adenoma or somatotropinoma (this category includes acromegaly and gigantism cases); PitNET NS, pituitary neuroendocrine tumor not specified; NF-PitNET, nonfunctioning PitNET; PRLoma, prolactinoma.

\section{Discussion}

We assessed the clinical value of genetic testing for AIP mutations with subsequent clinical screening of carriers in a cohort of patients with familial and young-onset PitNETs. In addition, we have compared the clinical features between AIPmut and AIPneg patients. Our key focus was on the follow-up of carriers and on prospectively diagnosed AIPmut patients, as the clinical and therapeutic characterization of this subgroup is lacking. The clinical screening of carrier family members of AIPmut probands has been recommended on the assumption that the early detection of PitNETs might be associated with more favorable outcomes $(1,3,13,14)$; however, these predicted advantages had not been previously demonstrated in a prospective study.

In the current study, among the 187 apparently unaffected AIPmut carriers, 22 were identified with a prospectively diagnosed PitNET by clinical, biochemical, and imaging screening. As a group, prospectively diagnosed AIPmut PitNETs were mainly microadenomas, smaller, and were associated with lower rates of suprasellar extension, cavernous sinus invasion, and hypopituitarism at diagnosis, and required fewer treatments, operations, no radiotherapy, and had reduced rates of active disease and hypopituitarism at last follow-up when compared with their clinically presenting counterparts. Similar results were obtained when prospectively diagnosed AIPmut somatotropinomas and AIPmut NF-PitNETs were analyzed separately. Overall, prospectively diagnosed AIPmut PitNETs are significantly less invasive and associated with better outcomes than those with a clinical presentation, highlighting the benefits of AIP genetic testing of family members at risk and the screening of individuals carrying an AIP mutation $(1,11,13,20)$.

In our series, 3 prospectively diagnosed PitNETs were not present at baseline assessment, but emerged during the follow-up (5-7 years after the initial screening), reinforcing the need for surveillance of unaffected AIPmut carriers $(1,3,13,14)$. These 3 cases are currently under observation, requiring no treatment. The AIPmut nonfunctioning microadenomas we have identified in our study are somewhat similar to the screening-detected MEN1-related pituitary tumors described elsewhere (21). In a different study, Tichomirowa et al. identified 2 patients with PitNETs among the 21 AIPmut carriers screened $(9.5 \%)$, both clinically silent microadenomas requiring no intervention (8). Both AIPmut and MEN1related prospectively diagnosed PitNETs should be managed in accordance with current guidelines (21-27).

There are 4 key questions for clinicians managing patients with PitNET regarding AIP: (1) Which clinically presenting pituitary tumor patients should be tested for AIP mutations? (ii) How to manage clinically presenting 


\section{Table 3. Comparative analysis between AIPmut vs AIPneg somatotropinomas}

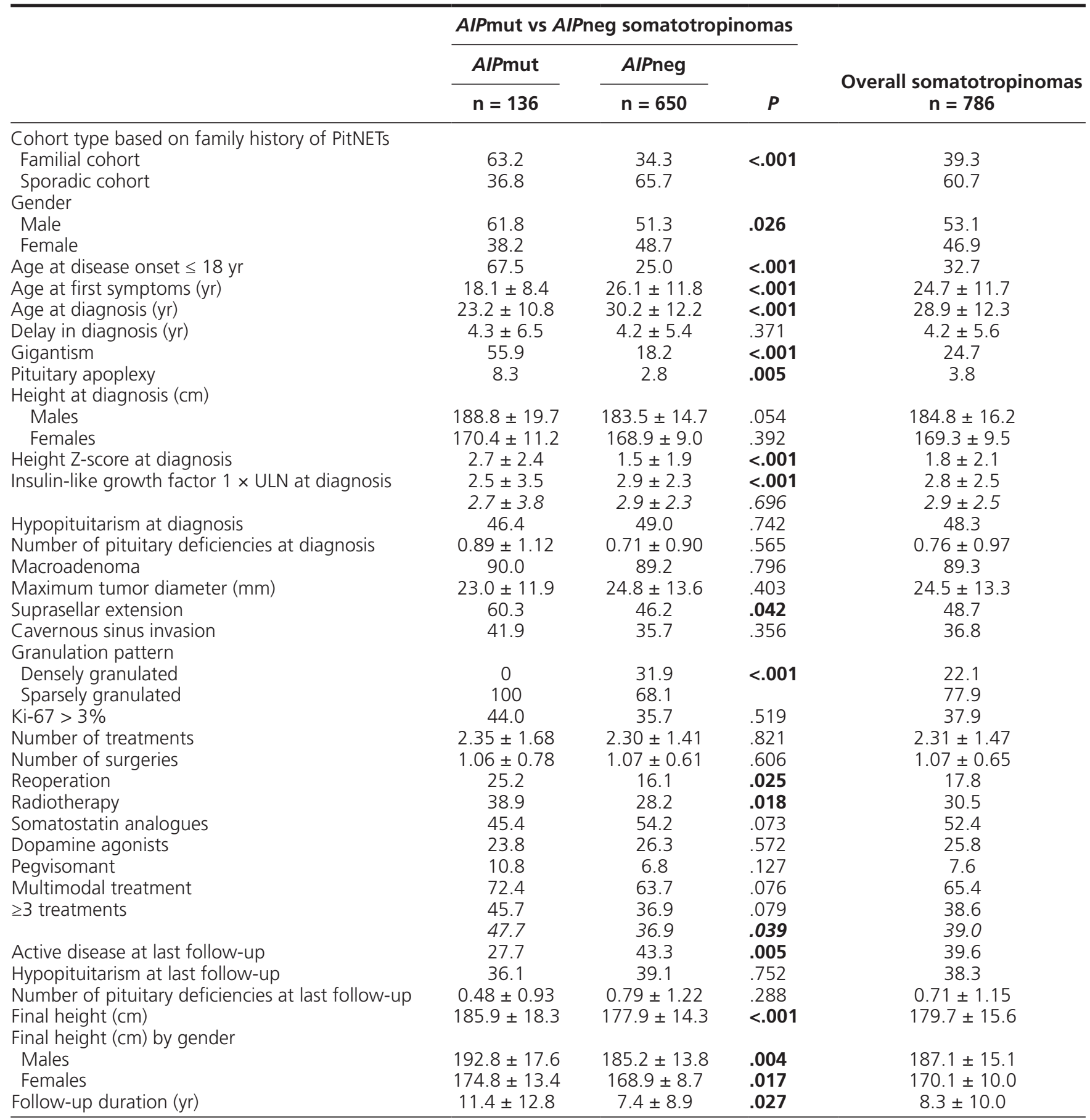

Categorical data are shown as \%; continuous variables are shown as mean \pm standard deviation. Data for clinically presenting somatotropinomas comparison are added in italics where showing different results. Data for clinically presenting somatotropinomas comparison are added in italics where showing different results. $P$ values in bold are those $<.05$ (statistically significant).

Abbreviations: AIPmut, AIP mutation-positive; AIPneg, AIP mutation-negative; PitNET, pituitary neuroendocrine tumor; ULN, upper limit of the normal; yr, years.

AIPmut PitNET patients? (3) When to initiate genetic screening for family members of a proband? and (4) What should be the clinical follow-up of AIPmut carriers?

1. Which clinically presenting pituitary tumor patient should be tested for AIP mutations? We have recently showed that based on 4 simple fac- tors (age of onset, family history, tumor type, and tumor size), the risk of carrying an AIP mutation can be predicted (11). As mutation status correlates with age of disease onset better than age of diagnosis (3), careful history taking is key. For example, age at onset between 19 and 30 years is an independent risk factor for patients with sporadic 


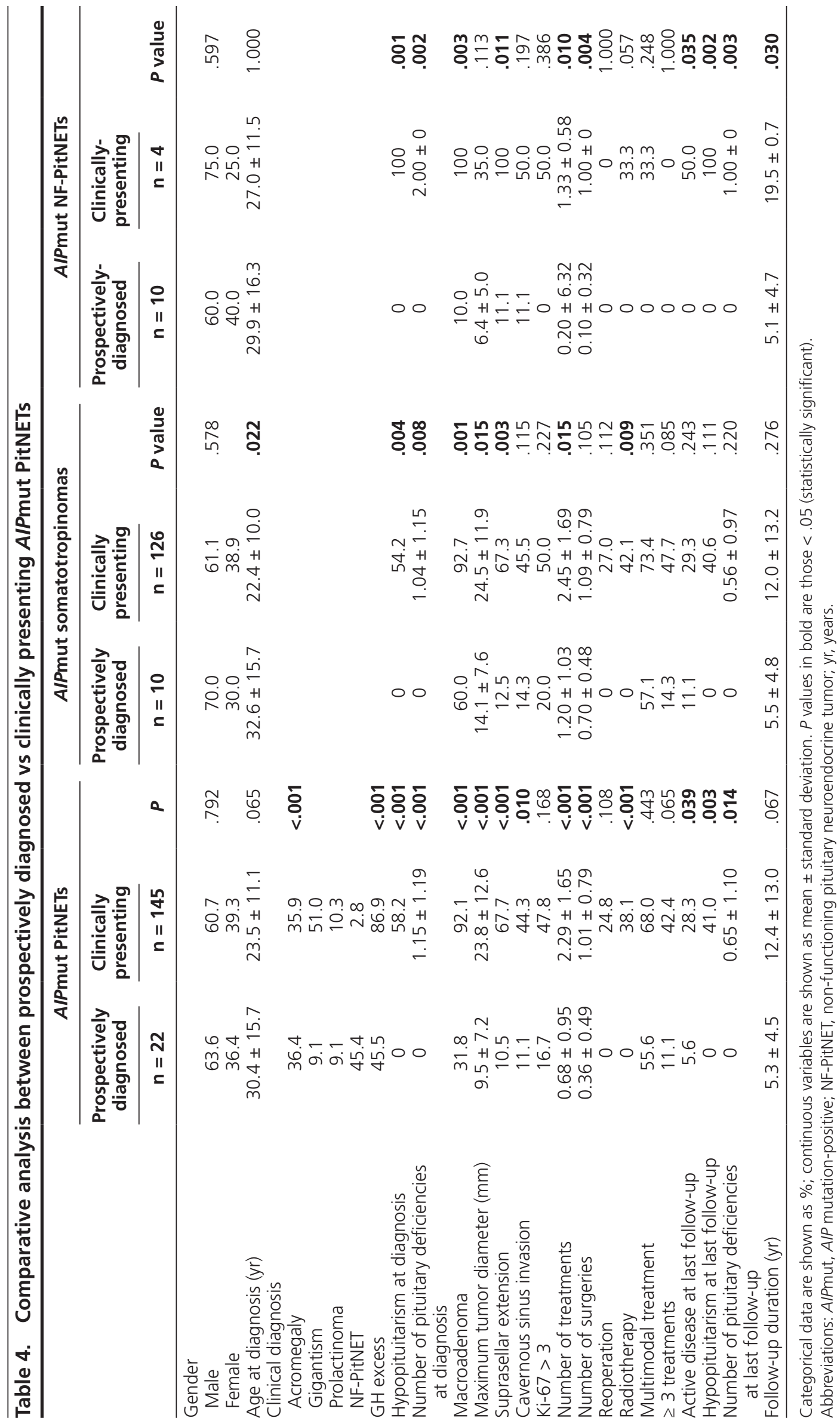


A)
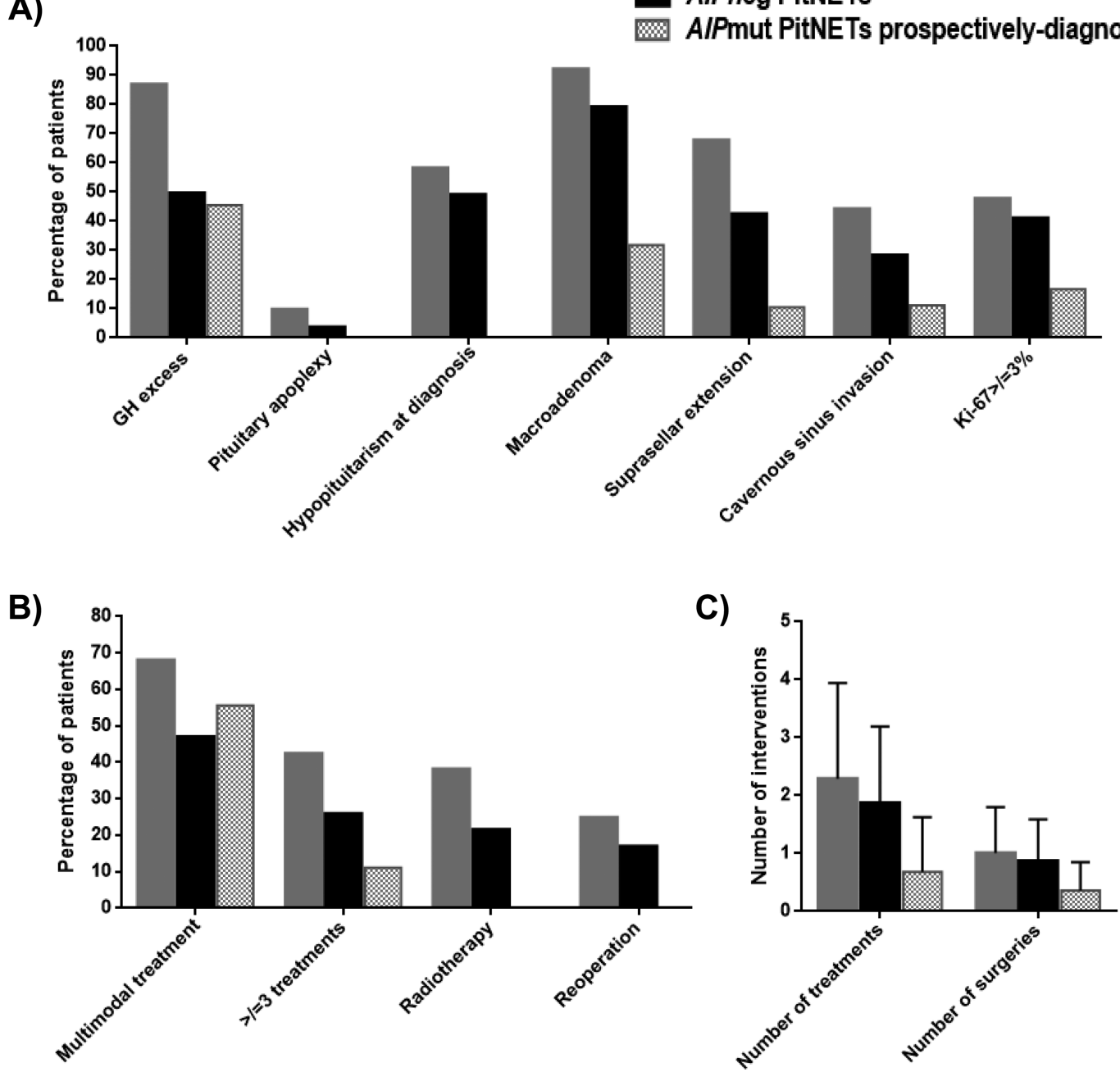

Figure 2. Patient characteristics $(A)$ and treatment modalities $(B, C)$. Clinical variables $(A)$ and treatment characteristics $(B, C)$ in patients with a clinically presenting PitNET, with or without AIP mutation (AIPmut and AIPneg), and in AIPmut carriers with an abnormality identified at clinical screening (prospectively diagnosed cases). (C) Data are shown as mean \pm standard deviation.

PitNET to carry an AIP mutation; however, patients in this age group without GH excess or an absence of family history have a lower risk (11). Hence, risk prediction should take several parameters into account, and for patients with fewer risk factors the age cut-off for AIP testing could be lower than 30 years $(11,28)$. Our fact-finding study shows that many patients with sporadic PitNET who undergo AIP analysis based on age at onset $\leq 30$ years $(3-5,11)$ will have negative results. In our young-onset sporadic PitNET cohort, $6.8 \%$ had an AIP mutation, with slightly higher rates in the sporadic somatotropinoma subgroup $(10.5 \%)$; this is at the level of usual risk recommendation for genetic testing, but we identified low rates in sporadic prolactinomas $(1.5 \%)$ with no cases of NF-PitNETs or corticotropinomas.
2. How to manage clinically presenting AIPmut PitNET patients? This is an important question but is largely beyond the scope of this article. There are numerous factors which need to be taken into account due to young onset, often aggressively growing tumors, and treatment should follow current guidelines, with attention to some characteristic features, such as aggressive growth, recurrence, poor response to firstgeneration somatostatin analogues with, at least in some cases, better responses to second-generation somatostatin analogues (29), and the risk of apoplexy. On the other hand, some cases show slower growth or stable nonfunctioning microadenomas, as shown in our data here.

3. When to initiate genetic screening for family members of a proband? We suggest germline AIP mutation genetic testing be offered at the earliest opportunity 
to first-degree relatives including children, because the disease may manifest by the age of 4 years (30).

4. What should be the clinical follow-up of AIPmut carriers? Our experience, based on this cohort, suggests that careful baseline assessment of AIPmut carriers (including clinical examination, measurement of serum IGF-1 and prolactin, and pituitary MRI) picks up the largest number of pituitary abnormalities. As AIP mutation testing has only been established just over a decade ago, the age range of establishing carrier status was very wide in our cohort. However, as testing is now routinely available, we predict that a larger number of carriers will be followed starting at an early age. As the age of disease onset has an inverted $\mathrm{U}$ shape (Fig. 1A), the recommendation for carrier follow-up could be different for the various age groups. For AIPmut carriers until the age of 20 years, annual clinical assessment with measurement of IGF-1 and prolactin and baseline MRI (starting at 10 years for younger carriers) followed by 5 -yearly scans could be appropriate. Follow-up between 21 and 30 years, if assessment is normal at age 20 years, probably could be relaxed. Our data also raise the possibility that adult AIPmut carriers with a normal baseline assessment could be followed with clinical and biochemical assessment, with further pituitary MRI only indicated in case of symptoms or biochemical abnormalities. Most clinically presenting cases show symptoms before the age of 30 years $(1,3)$, and we are not aware of any case with a normal full assessment at age $\geq 30$ years who later developed a PitNET. However, a cost-effectiveness analysis evaluating the economic burden of genetic testing and clinical screening programs in this setting, while weighing the benefits of early detection of AIPrelated pituitary disease we show in this study, is currently lacking.

In the AIPmut and AIPneg comparison, AIPmut PitNETs presented earlier with more aggressive disease and were more difficult to treat, as seen in previous studies $(3-5,10,31)$. Nevertheless, our data show that some AIPmut PitNETs will not display an aggressive phenotype $(4,8,12,32)$. Interestingly, the inclusion of aggressive or therapy resistant pituitary disease did not increase the frequency of AIP mutations in a recent study (28). Moreover, in our cohort, the rate of active disease at last follow-up was $10 \%$ lower in the AIPmut PitNETs group, suggesting that AIPmut PitNETs can be satisfactorily controlled despite requiring more complex and multimodal therapeutic schemes $(12,29,30,33,34)$. Although these data may seem paradoxical (more aggressive disease at presentation in the AIPmut patients, but better controlled disease at last follow-up), they could be explained by a more aggressive treatment approach in AIPmut cases, especially the use of radiotherapy. Another possibility is that the follow-up of AIPneg cases in our cohort was somewhat shorter; indeed, considering a cut-off of a maximum of 10 years of follow-up, there was no difference in rate of active disease between the 2 groups. Rostomyan et al. also reported higher rates of biochemical control at last follow-up and a trend for increased long-term controlled disease in patients with AIPmut pituitary gigantism in comparison to genetically negative gigantism cases (12). Thus, these data suggest that management of AIPmut patients can be challenging, but the disease is controllable in a significant proportion of cases.

Among AIPmut patients, somatotropinomas were the main PitNET subtype and gigantism the predominant clinical diagnosis, as previously shown $(4,11)$. IGF-1 levels at diagnosis did not differ between clinically presenting AIPmut and AIPneg somatotropinoma patients, suggesting that AIPmut somatotropinomas are not biochemically more active at presentation than their AIPneg counterparts, similar to earlier data (4). AIPmut patients with gigantism also showed similar IGF-1 levels in our cohort (35), although AIPneg giants had higher IGF-1 in another cohort (12). AIPmut somatotropinoma patients received radiotherapy more frequently than AIPneg patients, for which a nonsignificant trend had been observed previously (4). In addition, the mean final height in our cohort was higher in the AIPmut somatotropinoma subgroup, with both AIPmut males and females ending up taller than AIPneg counterparts, although this has not been consistently shown in other series (12). The taller final height in our AIPmut somatotropinoma patients is likely due to earlier onset of disease, but it may also reflect the management difficulties.

We found no differences regarding treatment and clinical outcomes in the comparative analysis of AIPmut vs AIPneg prolactinomas. Although numbers are small, this suggests that AIPmut prolactinomas may not be more refractory to medical therapy, in line with a previous report showing that presence of an AIP mutation in children or adolescents with macroprolactinomas does not influence the response to dopamine agonists (32).

AIPmut NF-PitNETs were smaller, had less pituitary deficiencies at diagnosis, and required fewer treatments and operations than AIPneg NF-PitNETs; however, these differences were lost when the 10 prospectively diagnosed cases were excluded from the analysis. In fact, clinically presenting AIPmut NF-PitNETs were macroadenomas, 
and had suprasellar extension and hypopituitarism at diagnosis/last follow-up, and half remain uncontrolled at last follow-up. Clinically presenting AIPmut NF-PitNETs reported previously were also noted for their aggressive behavior (4). Some of the small prospectively diagnosed AIPmut NF-PitNETs may represent incidentalomas similar to those often observed in the general population, although incidentalomas are more common in older subjects $(2,22)$. Prospectively diagnosed MEN1 mutation-positive NF-PitNETs also display an indolent behavior, do not progress to macroadenomas, and often require no intervention $(21,36)$. Overall, our data show that not all AIPmut PitNETs are aggressive or difficult to manage, as some patients have slowly growing or indolent NF-PitNETs (possibly representing incidentalomas) requiring no intervention, suggesting that the spectrum of $A I P$-related pituitary disease is wider than previously suggested.

Our study has some limitations: (1) we used the onset of symptoms age cut-off $\leq 30$ years as a criterion to guide AIP genetic testing in patients with young-onset sporadic PitNETs, as in previous AIP-related studies $(3-5,11)$. This age cut-off relies on age of onset, which can be subjective; however, age of onset rather than age at diagnosis is suggested to be a better option to guide genetic testing as PitNETs are often diagnosed with significant delay (11); (2) our patients were recruited from several countries and thus their clinical features and outcomes may be affected by their different genetic backgrounds and/or different local clinical practices; (3) we assigned, based on current experimental, clinical and in silico data, the AIP variants into pathogenic/likely pathogenic, or variant of uncertain significance/likely benign/benign groups; however, these categories may change as these variants are better characterized; (4) since the apparently unaffected participants of our study were genetically and clinically screened at various ages, we cannot determine, at this point, the disease penetrance for the prospectively diagnosed cohort per age group.

\section{Conclusions}

Genetic testing followed by clinical screening in AIPmut kindreds can detect clinically relevant pituitary disease, where earlier intervention results in better outcomes. While clinically presenting AIPmut PitNETs occur in younger patients with more advanced disease, complex treatment strategies can result in well-controlled disease. There is a wider spectrum of disease severity in AIPmut PitNET patients, even within the same family, than previously suspected. When considering patients for AIP mutation testing, key clinical factors help to predict the risk level to guide decision making.

\section{Acknowledgments}

The International FIPA Consortium includes the following: Prakash Abraham (Aberdeen, United Kingdom); Elena Aflorei (Manchester, United Kingdom); Amar Agha (Dublin, Republic of Ireland); James Ahlquist (Southend, United Kingdom); Scott A. Akker (London, United Kingdom); Krystallenia Alexandraki (Athens, Greece); Sándor Alföldi (Budapest, Hungary); João Anselmo (Ponta Delgada, Portugal); Wiebke Arlt (Birmingham, United Kingdom); Brew Atkinson (Belfast, United Kingdom); Anna Aulinas-Masó (Barcelona, Spain); Simon J. Aylwin (London, United Kingdom); Atik Baborie (Cardiff, United Kingdom); Philippe F. Backeljauw (Cincinnati, United States of America); Corin Badiu (Bucharest, Romania); Stephanie Baldeweg (London, United Kingdom); Steve Ball (Manchester, United Kingdom); Gul Bano (London, United Kingdom); Ariel Barkan (Michigan, United States of America); John Barton (Bristol, United Kingdom); Julian Barwell (Leicester, United Kingdom); Peter Bates (Jersey, United Kingdom); Carmen Bernal-González (Madrid, Spain); Michael Besser (London, United Kingdom); John S. Bevan (Aberdeen, United Kingdom); Alex Bickerton (Yeovil, United Kingdom); Jo Blair (Liverpool, United Kingdom); Marek Bolanowski (Wroclaw, Poland); Pierre Bouloux (London, United Kingdom); Lisa Bradley (Dublin, Republic of Ireland); Karin Bradley (Bristol, United Kingdom); Caroline Brain (London, United Kingdom); Antonia Brooke (Exeter, United Kingdom); Roger Brown (Edinburgh, United Kingdom); Michael Buchfelder (Erlangen, Germany); Christine Burren (Bristol, United Kingdom); Mehtap Cakir (Konya, Turkey); Natalie Canham (London, United Kingdom); Joel Capraro (Aarau, Switzerland); Paul Carroll (London, United Kingdom); Philippa Carter (Auckland, New Zealand); David Carty (Glasgow, United Kingdom); Dominic Cavlan (London, United Kingdom); Harvinder S. Chahal (London, United Kingdom); Tim Cheetham (Newcastle, United Kingdom); Farida Chentli (Algir, Algeria); Catherine Choong (Perth, Australia); Mirjam Christ-Crain (Basel, Switzerland); Teng-Teng Chung (London, United Kingdom); Peter Clayton (Manchester, United Kingdom); Richard N. Clayton (Stroke-on-Trent, United Kingdom); Mark Cohen (London, United Kingdom); Hamish Courtney (Belfast, United Kingdom); David Cove (Dorchester, UK); Elizabeth Crowne (Bristol, United Kingdom); Daniel Cuthbertson (Liverpool, United Kingdom); Jacob Dal (Aarhus, Denmark); Nadezhda Dalantaeva (Moscow, Russia); Svetozar Damjanovic (Belgrade, Serbia); Christina Daousi (Liverpool, United Kingdom); Ken Darzy (Stevenage, United Kingdom); Mehul Dattani (London, United Kingdom); Michaela Davies (Liverpool, United Kingdom); Justin Davies (Southampton, United Kingdom); Julian Davis (Manchester, United Kingdom); Margaret de Castro (São Paulo, Brazil); Laura de Marinis (Roma, Italy); Cheri Deal (Montreal, Canada); Judit Dénes (Budapest, Hungary); Paul Dimitri (Sheffield, United Kingdom); Neil Dorward (London, United Kingdom); Graham Dow (Nottingham, United Kingdom); William Drake (London, United Kingdom); Maralyn Druce (London, United Kingdom); Juliana Drummond (Belo Horizonte, Brazil); Pinaki Dutta 
(Chandigarh, India); Larisa Dzeranova (Moscow, Russia); Britt Edén-Engström (Stockholm, Sweden); Rosalind Eeles (London, United Kingdom); Maria Elfving (Lund, Sweden); Kate Ellis (Leicester, United Kingdom); Marianne Elston (Auckland, New Zealand); Louise Emmerson (Rhyl, United Kingdom); Shereen Ezzat (Toronto, Canada); Naomi Fersht (London, United Kingdom); Simona Fica (Bucharest, Romania); Stefan Fischli (Luzern, Switzerland); Maria Fleseriu (Portland, United States of America); Elizabeth Forsythe (London, United Kingdom); William Foulkes (Montreal, Canada); Pamela Freda (New York, United States of America); Theodore Friedman (Los Angeles, United States of America); Mónica Gadelha (Rio de Janeiro, Brazil); Mary Gainsborough (Bristol, United Kingdom); Stephen Gallacher (Glasgow, United Kingdom); Patricia Gallego (Brisbane, Australia); Hoong-Wei Gan (London, UK); Carmen Georgescu (Cluj-Napoca, Romania); Evelien Gevers (London, United Kingdom); Catherine Gilkes (Liverpool, United Kingdom); Nigel Glynn (London, United Kingdom); James E. Goldman (Boston, United States of America); Anthony P. Goldstone (London, United Kingdom); Miklós Góth (Budapest, Hungary); Andrew Green (Dublin, Republic of Ireland); Lynn Greenhalgh (Liverpool, United Kingdom); Joan Grieve (London, United Kingdom); Luiz Griz (Recife, Brazil); Mirtha Guitelman (Buenos Aires, Argentina); Alper Gürlek (Ankara, Turkey); Mark Gurnell (Cambridge, United Kingdom); Peter Shane Hamblin (Melbourne, Australia); Vaclav Hana (Prague, Czech Republic); Philip Harding (Adelaide, Australia); Eleanor Hay (London, United Kingdom); David A. Hilton (Plymouth, United Kingdom); Winnie Ho (Melbourne, Australia); Greg Hong (Charlottesville, United States of America); Katalin Horváth (Győr, Hungary); Simon Howell (Preston, United Kingdom); Trevor A. Howlett (Leicester, United Kingdom); Charlotte Höybye (Stockholm, Sweden); Steven Hunter (Belfast, United Kingdom); Chandi Idampitiya (Carlisle, United Kingdom); Péter Igaz (Budapest, Hungary); Ali Imran (Halifax, Australia); Warrick J. Inder (Brisbane, Australia); Takeo Iwata (Tokushima, Japan); Louise Izatt (London, United Kingdom); Sujatha Jagadeesh (Bombay, India); Colin Johnston (Watford, United Kingdom); Biju Jose (Stoke-on-Trent, United Kingdom); Gregory Kaltsas (Athens, Greece); Felicity Kaplan (Stevenage, United Kingdom); Niki Karavitaki (Birmingham, United Kingdom); Darko Kastelan (Zagreb, Croatia); Michelle Katz (Boston, United States of America); Tara Kearney (Manchester, United Kingdom); Melanie Kershaw (Birmingham, United Kingdom); Bernard Khoo (London, United Kingdom); Cathy Kiraly-Borri (Subiaco, Australia); Robertas Knispelis (Vilnius, Lithuania); Gábor L. Kovács (Budapest, Hungary); Anand Kumar (Madurai, India); Ajith V. Kumar (London, United Kingdom); Imre Zoltan Kun (Tirgu Mures, Romania); Angelos Kyriaku (Limassol, Cyprus); Ioana Lambrescu (Bucharest, Romania); Anne Katrin Lampe (Edinburgh, United Kingdom); Edward R. Laws (Boston, United States of America); Agnieszka Lebek-Szatanska (Warsaw, Poland); Ronald M. Lechan (Boston, United States of America); Graham Leese (Dundee, United Kingdom); Andrew Levy (Bristol, United Kingdom); Miles J. Levy (Leicester, United Kingdom); Krzysztof Lewandowski (Lodz, Poland); Eleanor Lin (Boston, USA); Janet Lo (Boston, United States of America);
Catherine Lyons (London, United Kingdom); Niki Maartens (Brisbane, Australia); Mohamad Maghnie (Genova, Italy); Taffy Makaya (Oxford, United Kingdom); Hani Marcus (London, UK); Marek Niedziela (Poznan, Poland); Niamh Martin (London, United Kingdom); Akira Matsuno (Chiba, Japan); Barbara McGowan (London, United Kingdom); Siobhán E. McQuaid (Dublin, Republic of Ireland); Milica MedicStojanoska (Novi Sad, Serbia); Nigel Mendoza (London, United Kingdom); Moisés Mercado-Atri (Mexico City, Mexico); Sachith Mettananda (Columbo, Sri Lanka); Emese Mezôsi (Pécs, Hungary); Dragana Miljic (Belgrade, Serbia); Karen K. Miller (Boston, United States of America); Silvia Modenesi (Belo Horizonte, Brazil); Mark E. Molitch (Chicago, United States of America); John Monson (London, United Kingdom); Damian G. Morris (Ipswich, United Kingdom); Patrick J. Morrison (Belfast, United Kingdom); Barbara Mosterman (Coventry, United Kingdom); Alia Munir (Sheffield, United Kingdom); Robert D. Murray (Leeds, United Kingdom); Madalina Musat (Bucharest, Romania); Nina Musolino (São Paulo, Brazil); Lisa Nachtigall (Boston, United States of America); Dinesh Nagi (Wakefield, United Kingdom); Ramesh Nair (London, United Kingdom); Richard Nelson (Bristol, United Kingdom); John Newell-Price (Sheffield, United Kingdom); Khash Nikookam (London, United Kingdom); Arla Ogilivie (London, United Kingdom); Steve M. Orme (Leeds, United Kingdom); Martin O’Weickert (Coventry, United Kingdom); Aparna Pal (Oxford, United Kingdom); Ionela Pascanu (Tirgu Mures, Romania); Attila Patócs (Budapest, Hungary); Catherine Patterson (Fife, United Kingdom); Simon H. Pearce (Newcastle, United Kingdom); Francesca Pecori Giraldi (Milan, Italy); Lynette Penney (Halifax, Canada); Luis Gustavo Perez-Rivas (Munich, Germany); Marija Pfeifer (Ljubljana, Slovenia); Fraser Pirie (Durban, South Africa); Nicola Poplawski (Adelaide, Australia); Vera Popovic (Belgrade, Serbia); Michael Powell (London, United Kingdom); Peter Pullan (Perth, Australia); Richard Quinton (Newcastle, United Kingdom); Serban Radian (Bucharest, Romania); Harpal Randeva (Warwick, United Kingdom); Narendra Reddy (Leicester, United Kingdom); Aled Rees (Cardiff, United Kingdom); Valerie Renals (Leicester, UK); António Ribeiro de Oliveira Jr. (Belo Horizonte, Brazil); Tristan Richardson (Bournemouth, United Kingdom); Celia Rodd (Montreal, Canada); Richard J. M. Ross (Sheffield, United Kingdom); Federico Roncaroli (Manchester, UK); Fiona Ryan (Oxford, United Kingdom); Roberto Salvatori (Baltimore, United States of America); Christof Schöfl (Erlangen, Germany); Debbie Shears (Oxford, United Kingdom); Kevin Shotliff (London, United Kingdom); Robert Skelly (Colchester, United Kingdom); Katie Snape (London, United Kingdom); Beatriz S. Soares (Belo Horizonte, Brazil); Noel Somasundaram (Colombo, Sri Lanka); Anna Spada (Milan, Italy); James Sperber (San Clemente, United States of America); Helen Spoudeas (London, United Kingdom); Maria Stelmachowska-Banas (Warsaw, Poland); Susan Stewart (Birmingham, United Kingdom); Helen L. Storr (London, United Kingdom); Christian Strasburger (Berlin, Germany); Maria Elisabeth Street (Parma, Italy); Isabelle Suter-Widmer (Luzern, Switzerland); Graeme Suthers (Adelaide, Australia); Francesca Swords (Norwich, United Kingdom); Luis V.Syro (Medellin, Colombia); Brede Swantje (Lübeck, Germany); 
Candy Sze (London, United Kingdom); Juliet Taylor (Auckland, New Zealand); Rajesh V. Thakker (Oxford, United Kingdom); Elaine Tham (Adelaide, Australia); Chris Thompson (Dublin, Republic of Ireland); Michael O. Thorner (Charlottesville, United States of America); Miklós Tóth (Budapest, Hungary); Peter J. Trainer (Manchester, United Kingdom); Stylianos Tsagarakis (Athens, Greece); Gina Twine (Plymouth, United Kingdom); Marinella Tzanela (Athens, Greece); Janos Vadasz (Szolnok, Hungary); Bijay Vaidya (Exeter, UK); Vladimir Vaks (Swindon, United Kingdom); Mary Lee Vance (Charlottesville, United States of America); Rasa Verkauskiene (Vilnius, Lithuania); Hilde Von Esch (Leuven, Belgium); John A. Wass (Oxford, United Kingdom); Mona Waterhouse (London, United Kingdom); Susan Webb (Barcelona, Spain); Astrid Weber (Liverpool, United Kingdom); Florian Wernig (London, United Kingdom); Hakan Widell (Gothenburg, Sweden); Shozo Yamada (Tokyo, Japan); Patrick Yap (Auckland, New Zealand); Sema Yarman (Istanbul, Turkey); Philip Yeoh (London, United Kingdom); Katsuhiko Yoshimoto (Tokushima, Japan); Kevin Yuen (Seattle, United States of America); Nicola N. Zammitt (Edinburgh, United Kingdom).

Financial Support: P.M. was supported by the Joan Adams and the Barts and The London Charity Clinical Research Training Fellowship. L.C.H.R. was supported by the Intramural Research Programs of Eunice Kennedy Shriver National Institute of Child Health \& Human Development. M.K. had funding from the Medical Research Council (UK), Rosetrees Trust and Pfizer to support the studies on familial isolated pituitary adenomas.

\section{Additional Information}

Correspondence and Reprint Requests: Márta Korbonits, Centre for Endocrinology, William Harvey Research Institute, Barts and the London School of Medicine and Dentistry, Queen Mary University of London, London, UK. E-mail: m.korbonits@qmul.ac.uk

Disclosure Summary: No potential conflict of interest.

\section{References}

1. Beckers A, Aaltonen LA, Daly AF, Karhu A. Familial isolated pituitary adenomas (FIPA) and the pituitary adenoma predisposition due to mutations in the aryl hydrocarbon receptor interacting protein (AIP) gene. Endocr Rev. 2013;34(2):239-277.

2. Molitch ME. Diagnosis and treatment of pituitary adenomas: a review. JAMA. 2017;317(5):516-524.

3. Hernández-Ramírez LC, Gabrovska P, Dénes J, et al.; International FIPA Consortium. Landscape of familial isolated and young-onset pituitary adenomas: prospective diagnosis in AIP mutation carriers. J Clin Endocrinol Metab. 2015;100(9):E1242-E1254.

4. Daly AF, Tichomirowa MA, Petrossians P, et al. Clinical characteristics and therapeutic responses in patients with germ-line AIP mutations and pituitary adenomas: an international collaborative study. J Clin Endocrinol Metab. 2010;95(11):E373-E383.

5. Cazabat L, Bouligand J, Salenave S, et al. Germline AIP mutations in apparently sporadic pituitary adenomas: prevalence in a prospective single-center cohort of 443 patients. J Clin Endocrinol Metab. 2012;97(4):E663-E670.

6. Cuny T, Pertuit M, Sahnoun-Fathallah M, et al. Genetic analysis in young patients with sporadic pituitary macroadenomas: besides AIP don't forget MEN1 genetic analysis. Eur J Endocrinol. 2013;168:533-541.

7. Preda V, Korbonits M, Cudlip S, Karavitaki N, Grossman AB. Low rate of germline AIP mutations in patients with apparently sporadic pituitary adenomas before the age of 40: a single-centre adult cohort. Eur J Endocrinol. 2014;171(5):659-666.

8. Tichomirowa MA, Barlier A, Daly AF, et al. High prevalence of AIP gene mutations following focused screening in young patients with sporadic pituitary macroadenomas. Eur J Endocrinol. 2011;165(4):509-515.

9. Vierimaa O, Georgitsi M, Lehtonen R, et al. Pituitary adenoma predisposition caused by germline mutations in the AIP gene. Science. 2006;312(5777):1228-1230.

10. Daly AF, Vanbellinghen JF, Khoo SK, et al. Aryl hydrocarbon receptor-interacting protein gene mutations in familial isolated pituitary adenomas: analysis in 73 families. J Clin Endocrinol Metab. 2007;92(5):1891-1896.

11. Caimari F, Hernández-Ramírez LC, Dang $\mathrm{MN}$, et al.; International FIPA consortium. Risk category system to identify pituitary adenoma patients with AIP mutations. J Med Genet. 2018;55(4):254-260.

12. Rostomyan L, Daly AF, Petrossians P, et al. Clinical and genetic characterization of pituitary gigantism: an international collaborative study in 208 patients. Endocr Relat Cancer. 2015;22(5):745-757.

13. Korbonits M, Storr H, Kumar AV. Familial pituitary adenomas who should be tested for AIP mutations? Clin Endocrinol (Oxf). 2012;77(3):351-356.

14. Marques P, Barry S, Ronaldson A, et al. Emergence of pituitary adenoma in a child during surveillance: clinical challenges and the family members' view in an AIP mutation-positive family. Int J Endocrinol. 2018;2018:8581626.

15. Williams F, Hunter S, Bradley L, et al. Clinical experience in the screening and management of a large kindred with familial isolated pituitary adenoma due to an aryl hydrocarbon receptor interacting protein (AIP) mutation. J Clin Endocrinol Metab. 2014;99(4):1122-1131.

16. Ellard S, Lango Allen H, De Franco E, et al. Improved genetic testing for monogenic diabetes using targeted next-generation sequencing. Diabetologia. 2013;56(9):1958-1963.

17. Marques P, Caimari F, Hernández-Ramírez L, et al. Data from: Significant benefits of AIP testing and clinical screening in familial isolated and young-onset pituitary tumors. Zenodo. 2020. Deposited 15 January 2020. doi: 10.5281/zenodo.3609165.

18. Richards S, Aziz N, Bale S, et al.; ACMG Laboratory Quality Assurance Committee. Standards and guidelines for the interpretation of sequence variants: a joint consensus recommendation of the American College of Medical Genetics and Genomics and the Association for Molecular Pathology. Genet Med. 2015;17(5):405-424.

19. Mothojakan NB, Ferrau F, Dang MN, et al. Polymorphism or mutation? - The role of the R304Q missense AIP mutation in the predisposition to pituitary adenoma. Paper presented at: Endocrine Abstracts Bioscientifica; November 2016, Brighton, UK, P167.

20. Daly AF, Beckers A. Familial isolated pituitary adenomas (FIPA) and mutations in the aryl hydrocarbon receptor interacting protein (AIP) gene. Endocrinol Metab Clin North Am. $2015 ; 44(1): 19-25$.

21. de Laat JM, Dekkers OM, Pieterman CR, et al. Long-term natural course of pituitary tumors in patients with MEN1: results from the DutchMEN1 Study Group (DMSG). J Clin Endocrinol Metab. 2015;100(9):3288-3296. 
22. Freda PU, Beckers AM, Katznelson L, et al.; Endocrine Society. Pituitary incidentaloma: an endocrine society clinical practice guideline. J Clin Endocrinol Metab. 2011;96(4):894-904.

23. Greenman Y, Stern N. Optimal management of non-functioning pituitary adenomas. Endocrine. 2015;50(1):51-55.

24. Katznelson L, Laws ER Jr, Melmed S, et al.; Endocrine Society. Acromegaly: an endocrine society clinical practice guideline. $J$ Clin Endocrinol Metab. 2014;99(11):3933-3951.

25. Nieman LK, Biller BM, Findling JW, et al.; Endocrine Society. Treatment of Cushing's syndrome: an endocrine society clinical practice guideline. J Clin Endocrinol Metab. 2015;100(8):2807-2831.

26. Blair J, Korbonits M, Ronaldson A, Dang M, Spoudeas H. National UK guidelines for managing pituitary adenomas in children and young people under 19 years developed according to the AGREE II framework. Paper presented at: International Symposium on Pediatric Neuro-Oncology; June 30 - July 3 2018, Denver, CO. CRAN-40.

27. Thakker RV, Newey PJ, Walls GV, et al.; Endocrine Society. Clinical practice guidelines for multiple endocrine neoplasia type 1 (MEN1). J Clin Endocrinol Metab. 2012;97(9):2990-3011.

28. Daly A, Cano DA, Venegas E, et al. AIP and MEN1 mutations and AIP immunohistochemistry in pituitary adenomas in a tertiary referral center. Endocr Connect. 2019;8(4):338-348.

29. Daly A, Rostomyan L, Betea D, et al. AIP-mutated acromegaly resistant to first-generation somatostatin analogs: long-term control with pasireotide LAR in two patients. Endocr Connect. 2019;8(4):367-377.

30. Dutta P, Reddy KS, Rai A, et al. Surgery, octreotide, temozolomide, bevacizumab, radiotherapy, and pegvisomant treatment of an AIP mutation-positive child. J Clin Endocrinol Metab. 2019;104(8):3539-3544.

31. Oriola J, Lucas T, Halperin I, et al. Germline mutations of AIP gene in somatotropinomas resistant to somatostatin analogues. Eur J Endocrinol. 2013;168(1):9-13.

32. Salenave S, Ancelle D, Bahougne T, et al. Macroprolactinomas in children and adolescents: factors associated with the response to treatment in 77 patients. J Clin Endocrinol Metab. 2015;100(3):1177-1186.

33. Mangupli R, Rostomyan L, Castermans E, et al. Combined treatment with octreotide LAR and pegvisomant in patients with pituitary gigantism: clinical evaluation and genetic screening. Pituitary. 2016;19(5):507-514.

34. Joshi K, Daly AF, Beckers A, Zacharin M. Resistant paediatric somatotropinomas due to AIP mutations: role of pegvisomant. Horm Res Paediatr. 2018;90(3):196-202.

35. Iacovazzo D, Caswell R, Bunce B, et al. Germline or somatic GPR101 duplication leads to X-linked acrogigantism: a clinicopathological and genetic study. Acta Neuropathol Commun. 2016;4(1):56.

36. Wu Y, Gao L, Guo X, et al. Pituitary adenomas in patients with multiple endocrine neoplasia type 1: a single-center experience in China. Pituitary. 2019;22(2):113-123. 